\title{
BLICKDIAGNOSE
}

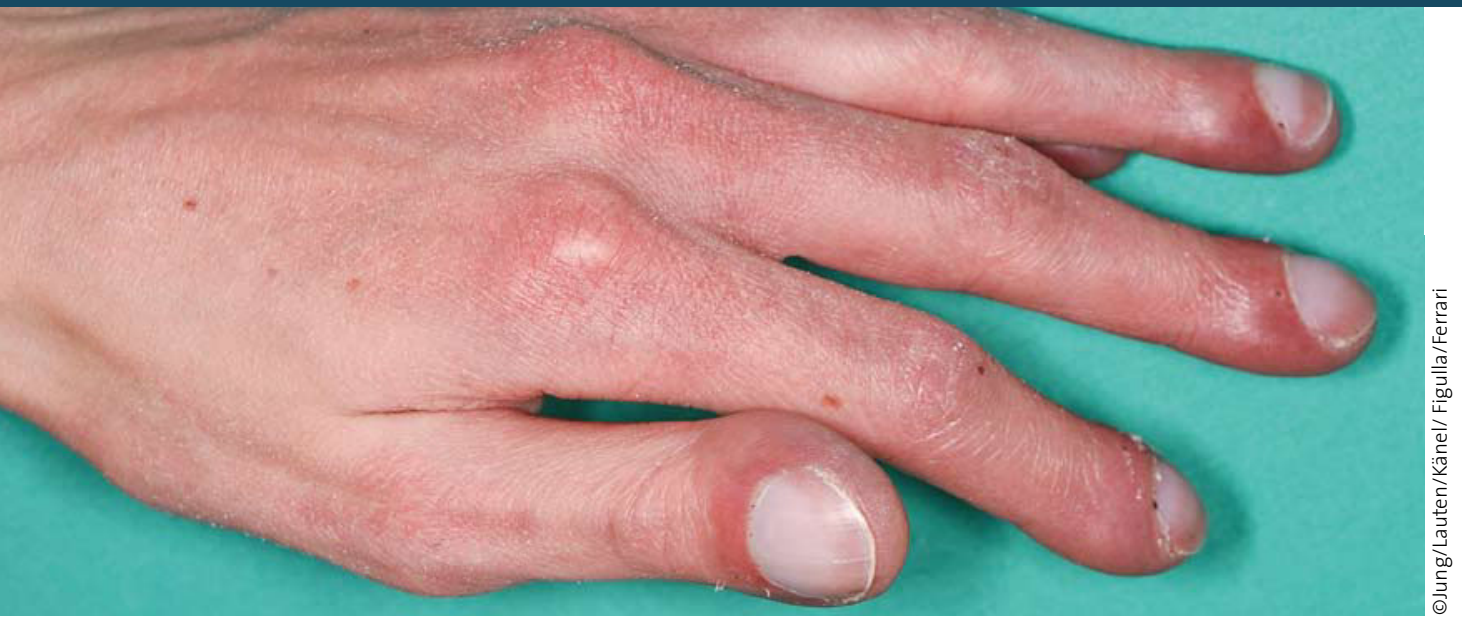

Die Finger des Hippokrates

\section{Zeigt her eure Hände!}

Das Phänomen der Trommelschlegelfinger wurde bereits um 400 vor Christus von Hippokrates beschrieben. Obwohl genaue Kriterien für die Trommelschlegelfinger und die häufig damit vergesellschafteten Uhrglasnägel definiert sind, handelt es sich zumeist um eine Blickdiagnose.

— Die Genese der Trommelschlegelfinger ist bis heute nicht abschließend geklärt. Neben der Hypothese, dass lokale arteriovenöse Fisteln eine pathophysiologische Rolle für mikrozirkulatorische Veränderungen spielen, wird eine andere Pathophysiologie aktuell favorisiert: Durch Veränderungen in der pulmonalen Strombahn, wie beispielsweise durch Entzündung, Tumoren oder auch durch intrakardiale Rechts-Links-Shunts, kann sie nicht - wie normalerweise vorgesehen - die aus dem Knochenmark freigesetzten Megakaryozyten in einzelne Thrombozyten fragmentieren. Die Megakaryozyten finden dann Eingang in die systemische Zirkulation und bleiben unter anderem in der Mikrozirkulation der Fingerspitzen hängen. Sie setzen dort Zytokine frei, die für pathophysiologische Veränderungen wie die Fibroblas- tenaktivierung etc. verantwortlich sind. Warum nicht alle Patienten mit diesen Erkrankungen Trommelschlegelfinger entwickeln und warum andere Krankheiten ohne Veränderungen in der Pulmonalstrombahn wiederum mit Trommelschlegelfingern einhergehen, bleibt bislang unklar.

Für den Kliniker ist es wichtig, bei der körperlichen Untersuchung die Trommelschlegelfinger wahrzunehmen und zu wissen, dass eine Reihe von Krankheiten damit vergesellschaftet ist, die es in der Folge weiter anamnestisch und differenzialdiagnostisch einzukreisen heißt. In Tabelle 1 sind die wichtigsten Differenzialdiagnosen aufgelistet. Unsere Abbildung zeigt Trommelschlegelfinger bei einem 22-jährigen Mann mit angeborenem Shuntvitium.

Keywords: drumstick fingers, Digiti hippocratici

- Dr. med. Christian Jung, Dr. med. Alexander Lauten, Dr. med. Joachim Känel, Prof. Dr. med. Hans-R. Figulla, PD Dr. Dr. med. Markus Ferrari, Klinik für Innere Medizin I der Universitätsklinik Jena, Erlanger Allee 101, D-07747 Jena

\section{Tabelle 1}

\section{Differenzialdiagnosen bei Trommelschlegelfingern}

Neoplasien Bronchialkarzinom, Pleuratumoren, Lymphom, nasopharyngeale Tumoren, Mesotheliom

Pulmonal Zystische Fibrose, Asbestose, Pneumonitis, interstitielle Fibrose, arteriovenöse Malformation in der Pulmonalstrombahn, Sarkoidose, Bronchiektasien, Lungenabszess

Kardial Zyanotischer Herzfehler (beispielsweise Fallot-Tetralogie, Transposition der großen Gefäße)

Gastrointestinal Chronisch entzündliche Darmerkrankung, Zöliakie, Leberzirrhose

Infektiös Tuberkulose, Endokarditis, verschiedene Parasiteninfektionen, HIV 